\title{
Marie-Angélique et la bride sur le cou : une critique sociale et politique dans Une fille d'Ėve de Balzac
}

\author{
Emilie M. Daniel Cersosimo \\ Escuela de Lenguas Modernas \\ Universidad de Costa Rica
}

\begin{abstract}
Résumé
Dans cette étude il s'agit d'une analyse de la contextualisation historique et sociale de la femme au XIXè siècle en France, dans le but de mieux comprendre le rôle de Marie-Angélique dans la société que Balzac décrit dans son roman Une Fille d'Ève. Les événements historiques, sociaux et littéraires influencent le rôle de la femme française de cette époque-là.
\end{abstract}

Mots clés: littérature française , femme, condition générique, histoire, société, morale, bourgeoisie, impositions, tâches, vie privée, Église, mariage, religion, mère, oppression, critique politique, critique littéraire, travail, féminisme, Code de Napoléon, roman réaliste, Comédie Humaine, Une Fille d'Ève, Honoré de Balzac, XIXè siècle

\section{Resumen}

Este estudio se trata de un análisis sobre la contextualización histórica y social de la mujer en el siglo XIX en Francia, con el fin de comprender mejor el papel de Marie-Angélique en la sociedad que Balzac describe en su novela Una hija de Eva. Los hechos históricos, sociales y literarios repercuten en el rol de la mujer francesa de aquella época.

Palabras claves: literatura francesa, mujer, condición de género, historia, sociedad, moral, burguesía, imposiciones, deberes, vida privada, Iglesia, matrimonio, religión, madre, opresión, crítica política, crítica literaria, trabajo, feminismo, novela realista, Código de Napoleón, Comedia Humana, Una hija de Eva, Honorato de Balzac, siglo XIX 


\section{La condition de la femme : historique, sociale, culturelle et générique}

$\mathrm{P}$ endant l'Ancien Régime en France, c'est-à-dire, la période de la Renaissance (XVIè siècle) à la Révolution française (1789), la femme

a été considérée comme nécessaire pour la société, puisqu'elle devait y jouer des rôles désignés par cette même société : la mère, l'épouse, la fille ou la religieuse. Depuis le Moyen Âge, elle était habituée à dépendre de l'homme et à l'obéir. Malgré cet état de subordination, il y en a eu qui ont pu travailler hors de la maison. Le sujet de la femme a été discuté depuis longtemps par des philosophes et des écrivains. Le XIXè siècle n'est pas l'exception, car l'inégalité sociale entre l'homme et la femme continuait (De Beauvoir, 1978 : 131-135).

Dans Une Fille d'Ève, nous trouvons Marie-Angélique, une femme qui a reçu, de même que sa sœur, une éducation très stricte du point de vue de la morale et de la religion conçue pour ne pas renoncer aux rôles imposés par la société patriarcale du XIXè siècle en France : «Angélique, disposée aux grandes luttes du sentiment, avait été jetée dans les plus hautes sphères de la société parisienne, la bride sur le cou ${ }^{1} »(29-30)$. Nous constatons, dans cet extrait du roman, que Marie-Angélique doit avoir le comportement adéquat d'une femme mariée et bourgeoise à Paris, et aussi elle est comparée à un cheval puisqu'elle portait " la bride sur le cou " et la bride est une pièce qui se fixe aux chevaux pour les diriger selon la volonté du cavalier. Par conséquent, cette bride pourrait symboliser la société qui dirige les actions de Marie-Angélique et qui contrôle sa conduite et ses actions. En plus, Marie-Angélique se montre comme une femme naïve, innocente et pure quand elle se marie avec Félix de Vandenesse; il faut ajouter que leur mariage n'a pas été par amour, mais il a été arrangé par la mère de Marie-Angélique :

Les mères de famille devraient rechercher de pareils hommes pour leurs filles : l'Esprit est protecteur comme la Divinité, le Désenchantement est perspicace comme un chirurgien, l'Expérience est prévoyante comme une mère. Ces trois sentiments sont les vertus théologales du mariage. (47)

Dans l'extrait ci-dessus, nous pouvons voir comment la mère de Marie-Angélique s'intéresse aux principes religieux par rapport au mariage de sa fille. Il nous semble essentiel de dire que dans Une Fille d'Ėve nous avons constaté des caractéristiques générales du portrait de Marie-Angélique: premièrement, elle est comparée à sa sœur Marie-Eugénie, car lorsque le narrateur fait référence à elles, plusieurs fois dans le récit, il dit: " les deux Marie ", puisque toutes les deux s'appellent comme ça. Cela peut être aussi une manière d'exprimer que tantôt Marie-Angélique, tantôt sa sœur, seraient des femmes très similaires, des femmes bourgeoises élevées par leur mère sous les principes de la morale et de la religion. Deuxièmement, lorsque le narrateur fait la description du portrait physique de Marie-Angélique, nous constatons la comparaison d'égalité dans quelques traits du corps : " la même taille, le même pied, la même main ... le même teint " (28). De plus, Marie-Angélique est comparée à Ève et sa vie 
au Paradis plusieurs fois dans le récit. Nous croyons que plus important que l'aspect physique de Marie-Angélique c'est son portrait moral ; c'est-à-dire, que le portrait de la protagoniste comporte des comparaisons et des critiques du point de vue de la morale, de la religion, de la politique, enfin de la société patriarcale de Balzac. De plus, nous allons prendre en considération des faits historiques et la société de la France du XIXè siècle pour mieux comprendre le rôle de Marie-Angélique dans ce milieu, car nous partageons l'avis de l'anthropologue mexicaine Marcela Lagarde sur la condition de la femme et ses rapports avec l'histoire, la politique, la culture et sa condition générique :

La condición de la mujer está constituida por el conjunto de relaciones de producción, de reproducción y por todas las demás relaciones vitales en que están inmersas las mujeres independientemente de su voluntad y de su conciencia, y por las formas en que participan en ellas; por las instituciones políticas y jurídicas que las contienen y las norman; y por las concepciones del mundo que las definen y las interpretan. Por esta razón, son categorías intercambiables condición de la mujer, condición histórica, condición social y cultural y condición genérica²... (Lagarde, 1997 : 78)

\section{L’inégalité sociale entre l'homme et la femme}

Au XIXè siècle, on considérait que la France était un pays plus moderne $\mathrm{du}$ point de vue social que les autres pays européens. Néanmoins, les grands hommes de la Révolution tels que Mirabeau, Danton et Robespierre étaient antiféministes. Ils suivaient les idées de Jean-Jacques Rousseau qui considère que les droits égalitaires de la révolution sont réservés aux hommes car la femme est subordonnée à l'homme par nature. Ils travaillent pour l'émancipation de l'homme mais pas pour celle de la femme (Borloz Soto, 2001 : 61).

De plus, ce n'est que dans le siècle de Balzac, juste après la Révolution Française, que l'on arrête de se questionner sur l'âme de la femme ou si celle-ci peut être considérée comme un être humain, car il y avait un débat commencé dans le passé par rapport à la condition de la femme (Fraisse, 1991 : 11). Geneviève Fraisse exprime dans une analyse de la situation historique de la femme qu'il paraît qu'avant le XIXe siècle, avoir une âme signifiait avoir un esprit, c'est-àdire, un esprit ingénieux, qui raisonne, comme celui des hommes (Fraisse, 1991 : 12). Cette auteure considère que l'histoire des textes peut nous aider à mieux comprendre l'histoire de la représentation de la différence des sexes (Fraisse, 1991 : 16), c'est-à-dire pourquoi il y a eu des préjugés à cause de la distinction des sexes. Par exemple, les femmes ne sont pas prises en considération en ce qui concerne leurs droits. Pourtant les juifs ont été émancipés le 27 septembre 1791 et l'esclavage a fini le 4 février 1794. Nonobstant, la condition des femmes reste la même malgré la Déclaration des droits de la femme et de la citoyenne d'Olympia de Gouges en 1791 (Borloz Soto, 2001 : 61) laquelle ne produit aucun effet dans la société française de la fin du XVIIIè et début du XIXè siècles. 
En outre, les nouvelles règles établies pour la femme dans le Code de Napoléon en 1804 ont ralenti son émancipation. Nous pouvons ainsi dire que c'est une sorte de recul par rapport à sa condition dans la société et d'un asservissement accru, lié à la naissance de la civilisation industrielle et capitaliste (BrahimiChapuis et Kuentzmann, $1976: 10$ ).

Le Code de Napoléon va consolider la domination du mari et le divorce, une des conquêtes de la Révolution Française, devient encore plus difficile à obtenir pour la femme (Nadeije Laneyrie - Dagen, 1996 : 514-515). En effet, dans Une Fille d'Ève, on ne voit aucune pensée de Marie-Angélique concernant le divorce ou la séparation de son mari. Le divorce est supprimé en 1826 et rétabli jusqu'en 1884. Parmi les nouvelles dispositions du Code Civil napoléonien, nous pouvons mentionner, tout d'abord, l'importance que Napoléon, héritier d'une révolution bourgeoise, attribue à la mère comme supérieure à l'épouse. Par conséquent, il est interdit d'interroger sur la paternité et la femme célibataire s'avère négativement signalée. De plus, tantôt les femmes mariées, tantôt les prostituées sont exclues de la catégorie de citoyennes. Les seules femmes qui possèdent les droits de citoyennes sont les célibataires, car celles qui ont été épousées doivent obéir à leurs maris et ce sont eux qui possèdent des droits sur elles. Un exemple de ceci, c'était que si la femme tombait dans l'adultère le mari avait la prérogative de l'envoyer en prison, de deux mois jusqu'à deux ans, et d'obtenir ainsi le divorce. Cependant, si le mari était l'adultère, il n'était puni qu'avec une amende s'il amenait sa concubine chez lui et c'était la seule façon pour l'épouse d'obtenir le divorce. En effet, nous pouvons constater que le Code de Napoléon octroyait du pouvoir et de l'autorité à l'homme, tandis que la femme restait complètement privée de liberté et sous l'oppression masculine (De Beauvoir, 1978 : 134-135).

\section{La bourgeoisie et la morale de Marie-Angélique}

Il convient de mentionner qu'à partir du XVIIIè siècle la bourgeoisie attribue une grande importance à la famille et condamne le sexe hors du mariage (Van Ussel, 1974 : 103). La bourgeoisie montre de cette manière un grand pouvoir sur les individus au XIXè siècle, notamment sur les femmes, dû aux valeurs morales qu'on exige à ce groupe social discipliné par l’homme: « La burguesía al postular que sus normas de comportamiento son las únicas acordes con la moral, es incapaz de comprender los valores de las demás clases, a las que cuelga el sambenito de la inmoralidad" (Van Ussel, 1974 : 17). Par conséquent, le bourgeois du XIXè siècle vivait sous l'oppression sociale de la morale et c'est pourquoi il y avait des gens qui menaient une vie double, c'est-à-dire, qu'ils devaient cacher certains comportements pour éviter d'être signalés et méprisés par la société (Van Ussel, $1974: 22$ ). Nous pouvons dire que c'est le cas dans Une Fille d'Ėve, puisque Raoul doit se rencontrer avec Marie-Angélique à la dérobée. Voici un exemple de ce qu'il devait faire pour pouvoir la voir : " Il lui fallait se trouver presque chaque jour à cheval au bois de Boulogne, entre deux et trois heures, dans la tenue du plus fainéant gentleman " (144). 
Marie-Angélique tombe amoureuse de Raoul; cependant, on peut dire que son éducation et ses principes religieux et moraux sont plus forts que le désir charnel, car Marie-Angélique et Raoul éprouvent de l'amour innocent et pur, malgré les pensées de cette femme :

Ainsi aucun amour ne fut, en fait, plus innocent ni plus pur que l'amour de Raoul et de Marie ; mais aucun ne fut plus emporté ni plus délicieux en pensée. La comtesse avait été prise par des idées dignes du temps de la chevalerie, mais complètement modernisés. (121)

Souvenons-nous que les moralistes du Moyen Âge avaient déjà condamné le plaisir sexuel et tout acte sexuel qui n'était pas destiné à la procréation. En 1550 , il y a eu aussi une variation de la perception de la sexualité de la part des autorités ecclésiastiques, des pédagogues et des moralistes. Nous voulons faire remarquer que pendant la période de la consolidation de la bourgeoisie le puritanisme et la bigoterie se sont intensifiés (Van Ussel, 1974: 40). C'est ainsi que la société bourgeoise du XIXè siècle décide le type de personnalité qu'il faut considérer comme "normale " ou " pathologique». L'individu bourgeois doit contrôler ses passions et ses instincts; avoir le contrôle est une condition nécessaire pour une meilleure efficacité au travail et une meilleure adaptation sociale (Van Ussel, 1974: 44).

Simone de Beauvoir explique, dans Le Deuxième Sexe, que la femme a été considérée historiquement comme un organisme sexué, c'est-à-dire, que la femme a toujours été importante aux yeux de la société en raison de sa biologie, puisqu'elle est capable de donner vie et c'est l'homme qui s'est approprié de la femme à travers l'histoire, depuis l'apparition de la famille patriarcale. Dans ce type de famille, la femme reste opprimée (De Beauvoir, 1978 : 67-69). En tenant compte de cette perspective sur l'oppression historique dont Beauvoir parle, nous essaierons d'interpréter le comportement de Marie-Angélique, selon le contexte social et les signes d'oppression qui l'accablent. D'ailleurs, Balzac fait référence à la morale de la France dans Une fille d'Ève : «...quel homme aurait vers la fin de 1828, prévu les étranges bouleversements que 1830 devait apporter dans l'état politique, dans les fortunes et dans la morale ${ }^{3}$ de la France ?" (10). D'après cette inquiétude de Balzac et la réalité historique du XIXè siècle français - où la femme est jugée par son comportement - nous pourrions dire que Marie-Angélique représente la femme qui vivait sous l'oppression sociale et politique aussi bien par le Code Civil de 1804, que par les préjugés moraux de la bourgeoisie.

\section{Des impositions sociales et historiques sur la femme}

Le Consulat - établi comme résultat du coup d'État du Général Napoléon Bonaparte - devient le premier empire (1804-1815), et plusieurs guerres politiques se déroulent pendant ces années. C'est une époque essentielle pour la femme, grâce aux lois instaurées dans le Code Civil de Napoléon où l'on 
déclare que : " la femme est propriété de son mari comme l'arbre à fruits du jardinier » (Laneyrie-Dagen, 1996 : 521). Nous considérons que ces règles rendent encore plus évidente la rigueur qu'on a exercée sur les femmes au début du XIXè siècle et que le personnage Marie-Angélique du roman de Balzac la supporte jour âpres jour.

$\mathrm{Au}$ XIXè siècle, le peuple français était formé par le prolétariat ouvrier et par une autre classe qui se consolide, c'est-à-dire, la bourgeoisie, dont l'essor constitue le phénomène social le plus important du siècle, dû aux conséquences sur la vie et la production littéraire : la petite bourgeoisie laborieuse d'artisans, de boutiquiers, d'employés, à côté d'une grande bourgeoisie de la banque et de l'industrie, parmi laquelle il y a de plus en plus des millionnaires, et entre les deux une bourgeoisie moyenne, disposant à la fois d'argent et désireuse de s'élever socialement (Desyeux-Sandor, 1995 : 28). Dans Une Fille d'Ève, il est évident que Marie-Angélique et sa sœur Marie-Eugénie appartiennent à la bourgeoisie moyenne et doivent se conduire comme de bonnes femmes selon leur classe sociale et l'éducation reçue. En plus, nous pouvons remarquer le ton critique de l'auteur à travers le narrateur quand il fait référence à l'éducation restreinte des filles influencée par la religion que la mère leur impose :

Élevées dans un sombre hôtel du Marais par une femme dévote et d'une intelligence étroite qui, pénétrée de ses devoirs, la phrase classique, avait accompli la première tâche d'une mère envers ses filles, Marie-Angélique et Marie-Eugénie atteignirent le moment de leur mariage, la première à vingt ans, la seconde à dix-sept, sans jamais être sorties de la zone domestique où planait le regard maternel. Jusqu'alors elles n'étaient allées à aucun spectacle, les églises de Paris furent leurs théâtres. Enfin leur éducation avait été aussi rigoureuse à l'hôtel de leur mère qu'elle aurait pu être dans un cloître ${ }^{4}$. (11)

Nous voyons dans le texte précédent des exemples qui rendent très évidente la situation de Marie-Angélique dans la société patriarcale du XIXè siècle en France. L'utilisation de certains mots qui font penser aux obligations selon le rôle féminin : la "tâche " d'une mère d'élever les filles ; ainsi que l'importance du mot " mariage " qui est interpreté comme le destin de la femme selon les règles sociales. Par rapport à l'éducation des filles, on peut dire que Balzac emploie le mot « sombre " pour comparer leur éducation, qu’il qualifie comme très "rigoureuse ", avec l'éducation dans un " cloître ». De plus, celle qui se charge de l'éducation de Marie-Angélique et de Marie Eugénie est leur mère, pourtant l'auteur la décrit comme une femme " dévote " et "d'une intelligence étroite ", ce qui expliquerait l'éducation si limitée des deux filles à cause de la religion et de l'ignorance de leur mère. C'est précisément à travers la forme littéraire que l'œil critique de Balzac observe sa société.

De plus, dans cet extrait du roman, le rôle de la femme que Balzac décrit coïncide avec la perspective de Simone de Beauvoir, qui signale le mariage et la maternité comme des impositions sociales et historiques sur la femme : « ...tout 
ce qu'on peut faire c'est l'enfermer dans des situations où la maternité est pour elle la seule issue; la loi ou les mœurs lui imposent le mariage... " (De Beauvoir, 1978 : 67-69).

\section{Marie-Angélique consacrée à la vie privée et aux tâches d'une «bonne femme bourgeoise »}

Au XIXè siècle, il existe une " littérature de civilité ", une sorte de création littéraire en vigueur du XVIè jusqu'au XVIIè siècles que la société accepte en général. Il s'agit des règles de comportement par rapport au contact physique avec les autres gens. D’après cela, il est interdit de s'embrasser, de baiser la main ou le pied et de s'agenouiller devant une dame. C'est une sorte de nouvelle pudeur du propre corps et du corps des autres (Ariès et Duby, 1985 : 11). Dans Une fille d'Ève, il y a un exemple de cette restriction corporelle quand Marie-Angélique accepte le bras de Raoul, mais en cachette : "Par une belle journée du mois d'avril, la comtesse accepta le bras de Nathan dans un endroit écarté ${ }^{5}$ du bois de Boulogne » (146).

Cette restriction, plus les préjugés de la bourgeoisie et de l'Église et celles du Code Civil, témoignent de l'œil observateur de la société française sur ses individus et particulièrement sur Marie-Angélique. Nous voyons dans l'extrait suivant le jugement social à propos de la nouvelle de l'amant de Marie-Angélique : "La nouvelle de la passion mutuelle de Raoul et madame de Vandenesse circula dans le monde pendant cette soirée, non sans exciter des réclamations et des incrédulités... » (132-133).

De plus, l'individu du XIXè siècle veut se protéger du regard des autres et la famille devient ainsi un refuge, le centre de l'espace privée (Ariès et Duby, 1985 : 8). Il est de la sorte fondamental de défendre ou d'améliorer le rôle social dans la communauté et surtout depuis les XVè et XVIè siècles, où l'allure devient très importante et l'honneur aussi. Défendre l'honneur symbolisait du prestige, car l'individu n'était pas ce qu'il était vraiment, mais surtout ce qu'il pouvait faire semblant d'être. La défense de l'honneur pouvait impliquer le duel ou la vengeance publique offensive (Ariès et Duby, 1985 : 8). D'après cela, nous pouvons comprendre le comportement de Marie-Angélique quand elle se rend compte de la réprobation de la société contre elle et Raoul: " La comtesse, mise en garde contre la médisance par madame Octave de Camps, avait compris l'importance de sa situation en face du monde, et la fit comprendre à Raoul " (133).

$\mathrm{Au}$ XIXè siècle, le rôle de la femme est réduit à la vie privée. Son activité principale est plutôt domestique : rester à la maison et accomplir la tâche d'épouse et de mère que l'Église et la société lui ont imposée :

La Contrarreforma amplió el campo de la vida privada femenina... Su Introducción a la vida devota tuvo honda influencia; basándose en ella y utilizando en particular las misiones, la práctica del ministerio sacerdotal desarrolló durante los siglos XVII y XVIII un nuevo modelo de 
devoción femenina hasta en los medios populares, para que se vivieran con más intensidad las grandes verdades de la religión cristiana. (Ariès et Duby, $1985: 27$ )

La femme doit être fidèle, avoir une bonne conduite, servir à son mari et à ses enfants et prendre soin d'eux : les nourrir, les élever, et surveiller leurs maladies. Elle doit servir mais elle gouverne la maison. C'est l'épouse qui gère la vie privée de la maison et le mari participe dans l'économie extérieure car c'est lui qui doit soutenir la famille économiquement (Ariès et Duby, 1985 : 19-23). De ce fait, la femme est celle qui surveille le foyer et la morale de la famille, et de ce point de vue, elle a un certain pouvoir, mais devient une victime de l'opinion publique et risque de s'exposer au scandale et de briser la loi du silence (Ariès et Duby, 1985 : 29). D'après cela, nous pouvons dire que parfois la femme devait cacher certaines situations dans son espace privé. Un exemple peut être la situation de Marie-Angélique qui maintient en secret son amour pour Raoul. Dans le récit, nous constatons l'importance sociale des noms et des positions sociales des fausses amies de Marie-Angélique qui l'encouragent à l'idée d'un amant. De plus, nous confirmons que ses amies veulent « quelque malheur» pour Marie-Angélique, afin qu'elle brise les règles de la morale :

Ses prétendues amies, qui dominaient leur réputation de toute la hauteur de leurs noms et de leurs positions, lui dessinèrent à plusieurs reprises la séduisante figure de l'amant, et lui jetèrent dans l'âme des paroles ardentes sur l'amour, le mot de l'énigme que la vie offre aux femmes, la grande passion. Quand la comtesse demandait naïvement en petit comité quelle différence il y avait entre un amant et un mari, jamais une des femmes qui souhaitaient quelque malheur ${ }^{6}$ à Vandenesse ne faillit à lui répondre de manière à piquer sa curiosité, à solliciter son imagination, à frapper son cœur, à intéresser son âme. (59)

Nous observons dans l'extrait ci-dessous comment l'auteur semble critiquer la société du XIXè siècle, laquelle attriste Marie-Angélique et sa sœur à cause des préjugés religieux :

La société de leur mère, loin de présenter quelques ressources à leur cœur ou de stimuler leur esprit, ne pouvait qu'assombrir leurs idées et contrister leurs sentiments ; car elle se composait de vieilles femmes droites, sèches, sans grâce, dont la conversation roulait sur les différences qui distinguaient les prédicateurs ou les directeurs de conscience, sur leurs petites indispositions et sur les événements religieux ${ }^{7}$ les plus imperceptibles pour la Quotidienne ou l'Ami de la religion. (14-15)

Il faut remarquer que la femme et l'homme ont été influencés par les préjugés religieux et sociaux à travers l'histoire. On veut ajouter que Poulain de la Barre, au XVIIè siècle, critique le fait que les individus deviennent des 
esclaves de l'opinion publique et des habitudes, et qu'ils n'approuvent que ce qui est conforme aux règles établies (Amorós, 1993 : 16).

\section{La critique sociale et politique de la France du XIXè siècle dans Une fille d'Ève}

Les différences sociales s'accroissent pendant le siècle de Balzac. Par exemple, parmi les trente-deux millions d'habitants de la France il n'y a que cent mille hommes ayant le droit de vote. La plupart du peuple français n'a aucun droit politique sous le royaume de Louis XVIII jusqu'en 1824 et pendant celui de Charles X de 1824 à 1830. Ce roi, voulant supprimer la liberté de presse, indisposait le peuple français. Cette année-là, une révolution des étudiants, des ouvriers et des bourgeois a obligé le roi à fuir. C'est ainsi que le Duc d'Orléans, un des cousins de Charles X, devient le nouveau roi sous le nom de Louis-Philippe Premier (Ariès et Duby, 1985 : 78-80).

En quelques décennies, les techniques de l'imprimerie évoluent et on passe de la fabrication d'imprimer à la main à la machine à imprimer, en 1820 en France, ce qui va faciliter l'accès à la littérature à beaucoup plus de gens et en particulier à la bourgeoisie, qui était peu cultivée. De plus, au début du XIXè siècle les livres coûtent très cher, seulement quelques- uns possèdent une bibliothèque privée, et il faut aller aux cabinets de lecture pour lire sur place ou emprunter le livre en payant. Grâce à la nouvelle vitesse de l'imprimerie, les livres deviennent plus faciles à acheter, car les prix diminuent et il y a de plus en plus des bibliothèques publiques en France (Desyeux-Sandor, 1995 : 30-32). Voilà comment, la prolifération de quotidiens, d'hebdomadaires, de revues, de brochures en tout genre devient, après 1830 et surtout après 1850 , un véritable phénomène de société (Desyeux-Sandor, 1995 : 30).

De 1830 à 1835, la liberté de presse existe malgré l'instabilité politique. Dans Une fille d'Ève, nous constatons l'importance des journaux pour la critique sociale et politique; voyons l'utilisation de l'adjectif " effroyable " : " À cette époque, une foule de journaux créés pour chaque nuance accusaient l'effroyable pêle-mêle politique...» (111).

En plus, une nouvelle révolte due à une crise économique, met fin au règne de Louis-Philippe Premier, et la Deuxième République commence en 1848 et va durer jusqu'en1851. Les notions de liberté et d'égalité s'ajoutent à la devise de la fraternité créée pendant la Révolution Française. Le neveu de Napoléon Premier dirige la France, c'est Louis-Napoléon Bonaparte. Quelques années plus tard, le 4 septembre 1870, une manifestation parisienne contre le régime de Napoléon III annonce sa chute. La liberté de presse a été pendant quelques années supprimée jusqu'en 1881. Les idées socialistes sont accueillies par la société française du XIXè siècle, surtout par l'influence de l'époque industrielle capitaliste. Les pensées de Marx qui expliquent l'inégalité parmi les hommes sont soutenues par un grand nombre de femmes dont George Sand ${ }^{8}$, écrivaine célèbre qui dénonce l'injustice et l'atrocité des lois du XIXè siècle qui handicapaient la femme dans 
la famille et la société (De Beauvoir, 1978 : 140-142). Pour les femmes du XIXè siècle, il n'y a que deux possibilités : l'amour et le mariage (Ariès et Duby, 1985: 65); le premier n'est pas toujours possible et le deuxième est plutôt un contrat basé sur des intérêts économiques ou sociaux, comme dans Le Contrat de $M a$ riage de Balzac, et aussi dans Une fille d'Ève, où Marie-Angélique ne s'est pas mariée avec Félix par amour.

George Sand est une femme qui a lutté pour un monde plus juste où les hommes et les femmes puissent avoir droit à l'éducation et à une bonne vie sans misère : "Il s'agit d'ouvrir un monde à tous les êtres qui composent l'humanité, qu'ils soient hommes ou femmes, ils doivent échapper à l'esclavage de la misère et de l'ignorance " (Laneyrie-Dagen, $1996: 595)$.

Le mouvement réformiste qui se développe au XIXè siècle en France va contribuer au féminisme car celui-ci cherche la justice et l'égalité ainsi pour les hommes que pour les femmes. Proudhon se présente comme un opposant à ce mouvement du moment où il considère que le lieu pour la femme est la maison et de plus, que la femme est inférieure à l'homme du point de vue physique, moral et intellectuel. Il soutient aussi que la femme doit rester dépendante de l'homme (De Beauvoir, 1978 : 141-142).

En ce qui concerne le domaine du travail, il devient nécessaire que la femme travaille, et grâce à la Révolution Industrielle l'histoire de la femme commence à changer très lentement. Le philosophe allemand Engels considérait que le sort de la femme était profondément lié à l'histoire de la propriété privée et au patriarcat (De Beauvoir, 1978 : 142-143), qui l'avaient rendue inférieure et dépendante de l'homme.

Malgré la possibilité de travailler pour quelques femmes, la plupart d'entre elles sont exploitées, car elles reçoivent des salaires plus bas que ceux des hommes, doivent travailler plus d'heures sans rien gagner et quelques unes sont victimes des abus de la part des employeurs. En outre, le travail de la femme est réglementé jusqu'en 1874 et ce n'est qu'en 1892 que la loi interdit le travail nocturne et établit la limite de la journée de travail dans les usines. Néanmoins, en 1893, une femme gagne la moitié du salaire d'un homme pour les mêmes heures de travail (De Beauvoir, 1978 : 147). Nous pouvons apercevoir l'inégalité qui persiste entre l'homme et la femme, malgré les lois concernant le domaine du travail.

Au XIXè siècle en France, il y a des femmes qui vivent attachées à la maison et au travail, surtout à cause des lois établies par Napoléon. Il y a par la suite un recul dans sa condition et la femme perd ainsi des droits qu'elle avait gagnés comme celui du divorce. En général, elle est mal payée pour son travail et n'a aucun droit dans la vie politique. Dans Une fille d'Ëve et dans le cas particulier de Marie-Angélique, elle représente la femme soumise, qui ne peut pas divorcer malgré son malheur et qui n'a non plus de droits dans le domaine politique.

Néanmoins, la Révolution Industrielle favorise le développement de la littérature, grâce à l'imprimerie, et aussi à l'alphabétisation et à la diffusion de la lecture (Ariès et Duby, $1985: 10$ ), ce qui va contribuer à marquer un changement dans la condition des femmes qui, pour la première fois depuis le Moyen 
Âge, commencent à lutter et à protester pour leurs droits. Quelques-unes écrivent dans des journaux et d'autres publient des romans, comme George Sand. Le féminisme apparaît comme une sorte de libération de la femme, dans le but de changer la domination historique de l'homme sur elle (Brahimi-Chapuis et Kuentzmann, $1976: 10-12$ ). La France a vu sept systèmes politiques entre 1800 et $1900^{9}$ : le Consulat, l'Empire, la Restauration, la Monarchie de Juillet, la Deuxième République, le Deuxième Empire et la Troisième République. Cela met en évidence l'extrême instabilité politique du pays. Cependant, l'écrivain acquiert un nouveau statut au XIXè siècle. D'ailleurs dans Une fille d'Ève, on critique la situation politique très instable de la France :

... Émile et Raoul analysèrent, les pieds sur les chenets, la situation politique de la France en 1834. De quel côté se trouvaient les meilleures chances de fortune ? Ils passèrent en revue les républicains purs, républicains à présidence, républicains sans république, constitutionnels sans dynastie, ministériels conservateurs, ministériels absolutistes ; puis la droite à concessions, la droite aristocratique, la droite légitimiste, henriquinquiste, et la droite carliste. (110)

\section{Le contexte littéraire au XIXè siècle français}

Il est remarquable que pendant cette période, une énorme richesse littéraire se produit, grâce au romantisme, au réalisme et au symbolisme. C'est Beaumarchais qui affirme en 1777 le droit de propriété des auteurs sur leurs œuvres, c'est-à-dire, que leur travail puisse leur permettre de vivre de la plume. Ainsi, après la Révolution, l'écrivain commence à devenir un citoyen indépendant (Desyeux-Sandor, 1995 : 33). Le romantisme marque le début du XIXè siècle et la lutte contre les défenseurs des notions classiques de la littérature et de l'art. En outre, les événements qui bouleversent la société française de 1789 à 1815 vont accentuer, dans la première moitié du XIXè siècle, un mouvement des canons classiques : " Le trait dominant depuis 1789 est celui qu'a montré Balzac : la volonté qui, a force de persévérance et d'intelligence, aboutit à une œuvre » (Becker, $1992:$ 31-32).

De plus, le romantisme devient un mouvement littéraire qui apporte de grands talents comme Lamartine, Vigny, Nerval, Musset et Hugo. Le roman historique prend un autre chemin avec Stendhal et Balzac, considéré aussi dans cette tendance. Le romantisme triomphe pendant la Restauration et la Monarchie de Juillet. L’imagination et la sensibilité sont essentielles pour le développement du lyrisme personnel. Le réalisme a lieu pendant le Deuxième Empire. Ce mouvement littéraire cherche à étudier les hommes selon leur comportement, leur ambiance et les théories sociales et psychologiques; c'est le contraire du romantisme. Le roman devient le genre favori des écrivains réalistes, parmi lesquels les plus célèbres : Flaubert, Stendhal, Zola, Sand, et l'auteur de notre roman d'étude : Honoré de Balzac (Lagarde et Michard, 1970 : 10-12). Les progrès de 
l'imprimerie, l'essor de la presse, l'apparition du feuilleton, de même que la demande d'un public élargi vont encourager le développement du roman (DesyeuxSandor, $1995: 30$ ).

Le symbolisme va se développer vers la fin du XIXè siècle et pendant la Troisième République. Ce mouvement littéraire s'intéresse à la valeur musicale et symbolique des mots. Nous voyons ainsi que la littérature du XIXè siècle offre une grande richesse et toute une galerie de portraits depuis la femme victime comme La Fantine, séduite et abandonnée dans Les Misérables de Victor Hugo, jusqu'à la femme héroïne comme Madame de Rênal dans Le Rouge et le Noir de Stendhal, dame d'élite et raffinée malgré l'ambiance grossière et de province de l'endroit où elle habite (Brahimi -Chapuis et Kuentzmann, 1976 : 11-12).

En effet, la littérature du XIXè siècle montre les différents visages de la femme en s'inspirant de la réalité de la société française. Il serait pertinent de dire que les sujets tirés de l'observation de la vie quotidienne étaient jusqu'à cette époque-là considérés bas, triviaux et la plupart du temps traités de façon satirique, comique, ou didactico-morale. Cependant, ces sujets commencent à être abordés d'un ton plutôt sérieux (Becker, 1992 : 32). Un exemple c'est que Balzac s'inspire de la réalité de son époque et surtout du sujet de la femme qui pouvait vivre dans le malheur en raison de sa condition de soumission et des préjugés religieux, politiques et sociaux du XIXè siècle qui l'accablent.

\section{Balzac : un observateur aigu de la réalité politique et religieuse de son époque}

Les faits historiques s'avèrent d'une grande importance pour l'œuvre de Balzac; d'ailleurs cet auteur l'avoue lui-même :

J'écris à la lueur de deux vérités éternelles : la religion, la monarchie, deux nécessités que les événements contemporains proclament et vers lesquelles tout écrivain de bon sens doit essayer de ramener notre pays. (Bellesort, 1946 : 175)

Nous voyons que cet écrivain prend en considération la situation politique et religieuse de son pays et essaie de dire des vérités comme lui-même l'exprime; il est contre le royaume de Louis-Philippe et contre les catholiques :

Il n'y cherchait pas davantage la faveur de la cour, car aucun romancier n'a plus vivement attaqué le gouvernement de Louis-Philippe qu'il considérait comme une déchéance de la royauté. Cependant ni les catholiques ni les royalistes de son temps ne lui en ont su gré ; et les autres ne paraissent pas lui en avoir tenu rigueur. (Bellesort, $1946: 175$ )

En 1902, Paul Bourget publie l'article « La politique de Balzac», dans lequel il signale qu'elle était née d'une vision réaliste de la nature sociale comme son 
catholicisme était né d'une vision réaliste de la nature morale, et qu'il avait été le prophète et le premier ouvrier de la rupture entre l'erreur révolutionnaire et les vérités scientifiques (Bellesort, 1946 : 175). D’après cela, nous prouvons encore une fois que Balzac est un observateur aigu de la réalité politique et religieuse de son époque. Voici un exemple de son intérêt à l'instabilité politique de son pays dans Une fille d'Ève :

Nous vivons à une époque, madame, où rien n'est sûr, lui dit-il. Les trônes s'élèvent et disparaissent en France avec une effrayante rapidité. Quinze ans font justice d'un grand empire, d'une monarchie et aussi d'une révolution. Personne n’oserait prendre sur lui de répondre de l'avenir. (218-219)

En outre, parmi quelques écrivains qui s'opposent aux convictions politiques, sociales et religieuses nous trouvons Rousseau, Stendhal, George Sand, Flaubert et, bien sûr, Balzac. En effet, dans La Comédie Humaine, il montre des éléments anarchistes; il considère que la religion peut modérer la pensée, qui livrée à elle-même et insubordonnée peut être une cause de ruine aussi bien pour les individus que pour les sociétés (Bellesort, 1946 : 178-181). De plus, il critique les dévotes, la monarchie constitutionnelle, la suprématie de l'argent (Bellesort, 1946 : 181-198) et aussi la subordination de la femme. D'ailleurs, Balzac est connu dans ses débuts comme le romancier des femmes, car "il avait commencé par mettre dans ses intérêts cette moitié du public très essentielle à gagner " (Bellesort, 1946 : 181-198). De cette manière, l'auteur d'Une fille d'Ève acquiert un grand public féminin, peut-être parce qu'il s'intéressait au sujet de la femme, parce qu'il voyait la femme en tant qu'individu avec des droits et non seulement comme un objet décoratif du mariage, ou parce que :

... il prolongeait pour elles, dans le roman, l'âge légal de l'amour. Ce n'était pas seulement la femme de trente ans dont il faisait parfois son héroïne, c'était aussi celle de quarante avec toute la jeunesse de cœur qu'elle a gardée et l'expression pathétique d'un visage où la passion " se réveille dans les plis de la douleur et les ruines de la mélancolie». (Bellesort, 1946 : 225)

D’après l'idée précédente, Balzac s’intéresse à montrer la situation de la femme en ce qui concerne l'amour et l'âge de l'amour, mais aussi ses souffrances. Balzac a déclaré « ...qu'il voulait la liberté de la jeune fille, c'est-à-dire que la jeune fille sût avant le mariage à quoi elle s'engageait..." (Bellesort, 1946 : 229-230). Cette idée de Balzac exprime certainement son intérêt pour la femme et nous pouvons dire que c'est probablement la raison pour laquelle cet écrivain du XIXè siècle a dédié un grand nombre de ses romans au sujet de la femme, et particulièrement à celui la femme dans le mariage.

Balzac appartient au mouvement réaliste du XIXè siècle et certains critiques comme Montalembert ont défini le réalisme comme " une influence mortelle " qui infecte (...) la littérature, l'art et jusqu'à la philosophie " (Becker, 1992 : 32). De cette manière, le réalisme a subi des connotations négatives; on 
disait qu'il évoquait la grossièreté, la vulgarité, l'obscénité et l'immoralité (Becker, 1992 : 32). Un exemple serait l'œuvre complète de Balzac, étant donné qu'à partir du XVIè siècle et jusqu' en 1938, Rome publie des "Index des livres prohibés " (Indices librorum prohibitorum), où l'on peut trouver des œuvres de Stendhal, Notre-Dame de Paris de Victor Hugo et la totalité des ouvrages de Balzac (http://www.systerofnight.net/religion/html/germaine/les_4_femmes_de_dieu. html). D'après cela, l'Église décide de cacher certaines œuvres littéraires et que celles de Balzac soient interdites par cette institution. Serait-ce parce que cet auteur a dévoilé la réalité de la subordination de la femme qui la faisait souffrir et éprouver des sentiments de culpabilité si elle ne respectait pas les lois imposées par le patriarcat et par l'Église?

Balzac est considéré l'un des créateurs du réalisme moderne, de même que Stendhal. Cependant, on dit que ce que Balzac apporte de plus c'est une volonté nouvelle de lucidité, d'explication et de discussion de la réalité historique et sociale de son temps, fondée sur une observation systématique (Becker, 1992 : 3839). D'ailleurs, Une fille d'Ève appartient aux Études des Mours de La Comédie Humaine où Balzac se propose :

... la volonté de ne pas raconter des faits imaginaires, mais de s'appuyer sur la vie réelle contemporaine, telle que se présente sous tous ses aspects et en tous lieux. Les réalistes l'approuveront d'avoir commencé par accumuler les observations et d'avoir été attentif non seulement à l'entourage de l'individu, mais aussi à l'interaction de celui-ci avec son milieu social. (Becker, 1992 : 40)

Nous pouvons dire que Balzac s'inspire de son contexte social et historique pour que son œuvre ait plus de ressemblance avec la réalité de son siècle, comme il disait : "...la mission de l'art n'était pas de copier la nature, mais d'en figurer le mouvement et la vie »(Bellesort, 1946 : 265).

La société du XIXè siècle semble très matérialiste et c'est pour cela que Balzac peint le culte pour l'argent qu'il y avait. C'est pourquoi il est possible de voir des hypocrites, des coquins, des arrivistes qui triomphent dans ses romans (Becker, 1992 : 78). En outre, on dit que les romanciers réalistes et naturalistes rendent évident un monde en pleine mutation économique où l'argent joue un rôle capital : " c'est la source de la considération et du pouvoir, il motive la plupart des attitudes et des conduites » (Becker, 1992 : 78).

De plus, les relations sociales, les lieux et les conduites sont placés sous le signe du masque, c'est à dire, qu'il s'agit d'un monde de l'imposture dans lequel chaque individu joue un rôle. C'est ainsi que les romanciers semblent dénoncer l'hypocrisie de la société qui se cache sous une apparence d'honnêteté. Ce serait donc le leitmotiv du roman réaliste et naturaliste dont le but est de dévoiler ce que le monde ne veut pas montrer (Becker, 1992 : 78).

Nous avons fait un parcours panoramique des faits historiques et sociaux du XIXè siècle afin de mieux déterminer le poids de l'image de Marie-Angélique dans Une fille d'Ève, étant donné qu'elle représente une femme opprimée de cette 
époque-là. Et encore une fois, nous voulons signaler l'importance de l'histoire pour le rôle de la femme dans une période déterminée, qui nous montre le progrès de l'humanité selon les rapports existants entre les hommes et les femmes :

El cambio de una época histórica puede determinarse por la actitud de progreso de la mujer ante la libertad, ya que es aquí, en la relación entre la mujer y el hombre, entre el débil y el fuerte, donde con mayor evidencia se acusa la victoria de la naturaleza humana sobre la brutalidad. El grado de la emancipación femenina constituye la pauta natural de la emancipación general. (Amorós, 1993 : 10)

De cette manière, nous pouvons dire donc que la situation sociale et historique de la femme a servi comme inspiration à Balzac, pour mieux peindre la société de son temps dans ses romans, puisqu'il fait dans ses récits une description de la société française depuis la Révolution jusqu'à la Restauration. En effet, dans La Comédie Humaine, nous reconnaissons des comportements, des raisons et de la vie publique de tous les individus, des hommes et des femmes de la société du XIXè siècle en France :

His Comédie Humaine is a description of French society, as it existed from the time of the Revolution to that of the Restoration. In this series of stories we find the author engaged in analyzing the manners, motives and external life of the French man and woman in all grades of society. (Epiphanious, 2011)

Selon la critique littéraire, Balzac dénonce que la destinée historique des femmes est le mariage et que la bourgeoisie la confine à la cuisine, au ménage, aux mœurs et que leur vie enfermée les empêche d'être indépendantes (De Beauvoir, 1978 : 137-138). En outre, Simone de Beauvoir explique que la femme arrive parfois à accepter d'être dominée par l'homme et à jouer les rôles traditionnels de mère et d'épouse :

On espère qu'ainsi dupées, séduites par la facilité de leur condition, elles accepteront le rôle de mère et de ménagère dans lequel on veut les confiner. Et le fait est que la plupart des femmes de la bourgeoisie capitulent. Comme leur éducation et leur situation parasitaire les mettent sous la dépendance de l'homme, elles n'osent pas même présenter de revendications... (De Beauvoir, 1978 : 138)

Par rapport à cela nous pouvons conclure que Marie-Angélique représente une femme bourgeoise élevée par sa mère, à l'aide des règles de la religion et de la morale de la société de Balzac. Elle se marie avec Félix et accepte son rôle de mère et d'épouse comme c'était le modèle traditionnel de féminité de l'époque. L'oppression historique et les règles établies dans le Code de Napoléon lui interdisent de transgresser la bonne conduite qu'une femme comme elle doit préserver : «Marie-Angélique éprouva précisément pour Félix le sentiment que Félix 
souhaite de lui inspirer : une amitié vraie, une reconnaissance bien sentie, un amour fraternel... Elle était mère, et bonne mère... " (48).

\section{Notes}

1 Les mots ont été mis en gras par l'auteure de cette recherche.

2 Les citations et idées de Marcela Lagarde employées dans cette étude ont été traduites en français par l'auteure de cet article. D'autres citations de Marcela Lagarde sont dans la langue originale. Les mots ont été mis en gras par l'auteure de cette recherche.

3 Le mot a été mis en gras par l'auteure de cette recherche.

$4 \quad$ Les mots ont été mis en gras par l'auteure de cette recherche.

5 Les mots ont été mis en gras par l'auteure de cette recherche.

6 Les mots ont été mis en gras par l'auteure de cette recherche.

7 Les mots ont été mis en gras par l'auteure de cette recherche.

8 Pseudonyme d'Aurore Dupin, qui décide d'utiliser un nom masculin pour ne pas montrer son identité féminine.

9 Deux empires (1803-1814; 1852-1870), trois monarchies (1815-1824; 1825-1830; 1830-1848), deux républiques (1848-1852; 1870), trois révolutions $(1830,1848,1871)$. Le Consulat et l'Empire (1800-1814), Le retour de la Monarchie (1815-1848), La seconde République (1848-1852, Le second Empire (1852-1870), La Illè République (1870-1940).

La France de la Restauration à l'Empire (1814-1852) http://www.espacefrancais.com/ histoire-de-la-france-au-xixè-siècle

\section{Bibliographie}

AMORÓs Celia, in Poulain de la Barre, De la Educación de las Damas para la formación del espíritu en las ciencias y en las costumbres, Madrid, Ediciones Cátedra, 1993.

ARIÈS Philippe et Georges DUBY, Historia de la vida privada, $\mathrm{M}^{\mathrm{a}}$ Concepción Martín Moreno (trad.), Tomes 5 et 6, Paris, Éditions du Seuil, 1985.

BALZAC Honoré de, Euvres Complètes illustrées d'Honoré de Balzac, Tome premier, Tome deuxième, Tome troisième, Paris, Guy le Prat, 1960.

BECKER Colette, Lire le Réalisme et le Naturalisme, Paris, Dunod, 1992.

BELLESORT André, Balzac et son œuvre, Paris, Librairie Académique Perrin, 1946.

BORLOZ SOTO Virginia, «Madame Bovary soy yo» Flaubert y la Literatura Costarricense, San José, EUNED Editorial Universidad Estatal a Distancia, 2001.

BRAHIMI -CHAPUIS Denise et Lucile KUENTZMANN, Images de la femme, Poitiers, Éditions Delgrave, 1976.

DE BEAUVOIR Simone, Le Deuxième Sexe, Paris, Éditions Gallimard, 1949.

DESYEUX SANDOR Monique et Martine DAL ZOTTO, Anthologie de la littérature française XIXè siècle, Paris, Librairie Générale Française, 1995. 
FRAISSE Geneviève, Musa de la razón: La democracia excluyente y la diferencia de los sexos, Alicia H. Puleo (trad.), Madrid, Ediciones Cátedra, 1991.

LAGARDE Marcela, Los cautiverios de las mujeres madre esposas, monjas, putas, presas y locas, México, Universidad Nacional Autónoma de México, 3 ème édition, 1997.

LANEYRIE-DAGEN Nadeije, Mémoire de la France des origines à l'an 2000, Paris, Larousse, 1996.

VAN USSEL Jos, La Represión Sexual, J.A. Bravo (trad.), México, 1974.

\section{Documents électroniques :}

BALZAC, Honoré de, Une fille d'Ève, La Bibliothèque électronique du Québec, Collection À tous les vents, volume 810 : version 1.0, propriété exclusive de Jean-Yves Dupuis. beq.ebooksgratuits.com/balzac/Balzac-12.pdf

BALZAC, Avant-propos de La Comédie Humaine le premier de l'édition Furne, volume 1 (1842), disponible à http://books.google.com/) 28 novembre 2010. http://www.espacefrancais.com/histoire-de-la-france-au-xixè-siècle http://www.systerofnight.net/religion/html/germaine/les_4_femmes_de_dieu. html). 
